\title{
Politische Ursachen für Rentenprobleme und Altersarmut erfordern grundlegende Reformen
}

\author{
Die Große Koalition stellt mit der Grundrente vor allem langjährige Beitragszahler besser. \\ Allerdings bekämpft die Grundrente lediglich ein Symptom, statt sich den Ursachen zu widmen: \\ der Politik niedriger Löhne und falscher politischer Maßnahmen. Niedrige Löhne führen zu \\ niedrigen Renten. Die politische Verantwortung für niedrige Renten und Altersarmut macht zur \\ Erreichung existenzsichernder Renten einen steuerfinanzierten Ausgleich notwendig.
}

Das Deutsche Institut für Wirtschaftsforschung (DIW) erwartet eine wachsende Altersarmut bis zum Ende der 2030er Jahre (Geyer, 2019). Die Grundrente ändere daran nichts, weil sie nicht zielgenau sei. Das bedeutet, dass das Rentensystem nicht einmal seinen beabsichtigten Nebeneffekt „Vermeidung von Altersarmut“ erreicht. Die gesetzliche Rente verfolgte seit der Reform von 1957 und bis zu den Reformen 1999 das Ziel, die Arbeitseinkommen zu ersetzen, die mit dem Ende der Erwerbstätigkeit entfallen (Althammer und Lampert, 2014, 287; Schmähl, 2012, 306 ff.). Die Rente sollte - wenigstens tendenziell - „ein den Lebensstandard sicherndes Rentenniveau gewährleisten“ (Althammer und Lampert, 2014, 299). Die Armutsvermeidung war deshalb nur ein Nebenziel des deutschen Rentensystems. Und selbst dieses untergeordnete Ziel wird mit der neuen Grundrente nicht erreicht, wenngleich sie für einen Teil der Rentner Verbesserungen bringt. Dass das deutsche Rentensystem weder den Lebensstandard sichern noch Altersarmut vermeiden kann, ist wesentlich auf wirtschafts- und sozialpolitische Entscheidungen zurückzuführen, die in der bisherigen Diskussion keine Rolle spielten. Die erforderlichen Reformmaßnahmen müssen die politisch zu verantwortenden Ursachen aber berücksichtigen. Nun müssen Sonderlösungen im Rentensystem gefunden werden. Politisch sind drei entscheidende Fehler gemacht worden:

Erstens wurde mit den Riester-Reformen das Ziel des Lohnersatzes explizit und sachwidrig durch das Ziel der Beitragssatzstabilität ersetzt (Althammer und Lampert, 2014), was zwangsläufig eine Absenkung des Leistungsniveaus der gesetzlichen Rentenversicherung zur Folge hatte. Die Begründung dafür, dass wegen der demografischen Entwicklung der Unterhalt des steigenden Rentner-

(C) Der/die Autor(en) 2020. Open Access: Dieser Artikel wird unter der Creative Commons Namensnennung 4.0 International Lizenz (https:// creativecommons.org/licenses/by/4.0/deed.de) veröffentlicht.

Open Access wird durch die ZBW - Leibniz-Informationszentrum Wirtschaft gefördert. anteils die junge Generation überfordere, ließ außer Acht, dass auch Produktivität und Realeinkommen steigen werden und dass das Nettorealeinkommen der jungen Generation nach Abzug höherer Beiträge selbst bei moderater jährlicher Steigerung höher ausfallen wird als das der gegenwärtigen Erwerbsgeneration. Die Behauptung der Überforderung der jungen Generation ist aber auch schon deshalb nicht haltbar, weil die reformbedingte Rentenlücke in der gesetzlichen Versicherung - soll das Sicherungsniveau erhalten bleiben - durch Aufwendungen für private Versicherungen zu decken wäre, die wegen der höheren Kosten privater Versicherungen höher ausfallen als die Beiträge zur gesetzlichen Versicherung. Darüber hinaus belastet die Deckelung des Beitragsanteils der Arbeitgeber die Beitragszahler zusätzlich. Es ist also umgekehrt: Das Rentennvieau wird trotz öffentlicher Förderung eine höhere Belastung der jungen Generation zur Folge haben als normal steigende Beiträge in die gesetzliche Rentenversicherung sie zur Folge hätten. ${ }^{1}$ Die geplante Schließung der Lücke wird von der Mehrheit der Betroffenen gar nicht genutzt. Sie konnte schon wegen der höheren Kosten privater Versicherungen nicht gelingen. Erforderlich ist der Ausstieg aus der Riester-Reform.

Zweitens wurde eine Wirtschaftspolitik betrieben, die das Lohnniveau niedrig hielt. Das geschah durch eine „moderate“ Lohnpolitik (Bofinger, 2005; Hickel, 2006; Flassbeck

1 „In dem nun propagierten ,Mehrsäulensystem“ wird es für die Bürger teurer, ein dem Leistungsniveau der GRV vergleichbares Niveau zu finanzieren - vor allem auch für junge Menschen, obgleich angeblich in deren Interesse die Umstrukturierung dringend notwendig war" (Schmähl, 2016).

Dr. Ernst Niemeier war Lehrbeauftragter an der Hochschule Bremen. 
und Spieker, 2007), die die Produktivitätssteigerungen nicht ausschöpfte und so die Binnennachfrage schwächte. Später vergrößerte die Agendapolitik den Niedriglohnsektor. Die behauptete mangelnde Wettbewerbsfähigkeit wird durch die vergleichsweise geringe deutsche Lohnstückkostenentwicklung ${ }^{2}$ und die deutschen Exportüberschüsse widerlegt. ${ }^{3}$

Drittens wurden über viele Jahre eine restriktive Geldpolitik (Wyplosz, 2007, 88 f.) und eine „systematisch prozyklische“" Fiskalpolitik (Wyplosz, 2007, 91 f.; Niemeier, 2009) betrieben. Die folgende Nachfrageschwäche senkte die Wirtschaftsdynamik, beeinträchtigte die Produktion und verhinderte ein ausreichendes Arbeitsplatzangebot.

\section{Äquivalenzprinzip setzt die Leistung voraus}

Müssen Lösungen für diese Probleme das Äquivalenzprinzip einhalten? Die Rentenhöhe ergibt sich gemäß diesem Prinzip aus der Höhe der einkommensabhängigen Beitragszahlungen. Die negativen politischen Einflüsse auf die Möglichkeiten des Einkommenserwerbs stellen in einem Rentensystem, das auf dem Äquivalenzprinzip beruht, ein Sonderproblem dar. Der zwischen Erwerbseinkommen und Rente bestehende systematische Zusammenhang (Scherf, 2011, 197) stellt eine fundamentale Gerechtigkeitsnorm dar (Scherf, 2011, 193). Die Rentenleistung setzt aber eine vom Versicherten verantwortete, „echte“ Leistung voraus. Wenn Einkommen und Beitragsleistung durch staatlich verursachte Blockaden vermindert werden, dann bewirkt das Äquivalenzprinzip eine zu geringe Rente; dann darf das Äquivalenzprinzip nicht unbesehen angewandt werden. Die Beitragsleistung eines Versicherten, der entgegen seinen Fähigkeiten und Wünschen gezwungen ist, einen Niedriglohn zu beziehen, darf nicht mit den Folgen dieser Politik bestraft werden. Stattdessen muss die Gesellschaft als politischer Verursacher der Einkommensminderung für den verursachten Schaden aufkommen. Die Rente muss in diesen Fällen durch angemessene steuerlich finanzierte Ausgleichsleistungen aufgestockt werden. Eine Finanzierung aus Beiträgen und auch eine Bedürftigkeitsprüfung ist verursachungsgemäß auszuschließen.

In der Grundrentendiskussion wurde diese Verantwortung nicht beachtet, weil die vorherrschende Meinung in der deutschen Ökonomie die fehlerhafte Politik nicht erkennt. Sie sah die Ursachen für die wirtschaftlichen Schwierigkeiten irrtümlich oder interessengeleitet einseitig auf der Angebotsseite, also bei Beschäftigten und Löhnen. Die

2 Horn kommentiert dazu: „In den vergangenen 24 Jahren sind in keinem anderen Industrieland mit Ausnahme Japans, ..., die Lohnstückkosten so geringfügig gestiegen wie in Deutschland“ (Horn, 2005, 120).

3 So wies Solow (2007, 40 f.) darauf hin, dass „ein Exportüberschuss normalerweise kein Zeichen überhöhter Löhne" sei.
Lohnstückkosten und die deutschen Exportüberschüsse weisen jedoch nicht auf angebotsseitige Probleme hin. Im Gegenteil, die schwache Binnennachfrage zeigt, dass die Ursache für die lahme Wirtschaftsentwicklung mit der Folge prekärer Beschäftigungsverhältnisse und mit Arbeitslosigkeitszeiten auf der Nachfrageseite lag. Die deutsche Politik hatte die Nachfrageseite nicht im Blick. Durch die einseitige Ausrichtung auf das Angebot, durch Lohnmoderation und Unterstützungskürzungen, wurde die gesamtwirtschaftliche Nachfrage zusätzlich geschwächt. Horn $(2007,117)$ wies empirisch nach, dass die Ursachenindikatoren „in Richtung Nachfrageproblem weisen“. Deshalb sei der „grundlegende Fehler der aktuellen Wirtschaftspolitik in Deutschland strategischer Natur, da sie auf der ,falschen' Seite des Marktes angesetzt hat". Solow $(2007,40)$ bestätigte diese Erkenntnis: es gebe gute Gründe für die Annahme, „dass ein bedeutender Teil der deutschen Arbeitslosigkeit einen Okun Gap (Nachfragelücke) widerspiegelt und auf eine expansive Nachfragepolitik reagieren würde. Es scheint mir ein intellektueller Fehler zu sein, dass diese Seite des Problems wenig oder gar keine Bedeutung in der politischen Debatte erhält". Auch die Agendapolitik, die ebenfalls auf die Angebotsseite gerichtet war, schwächte die Nachfrage und verantwortet die Rentenkrise mit.

\section{Agenda weitete Niedriglohnsektor aus}

Die Agendapolitik sah die Ursache für die bestehende hohe Arbeitslosigkeit in zu hohen Löhnen und der Arbeitsunwilligkeit von Arbeitslosen. Der damalige Bundeskanzler Schröder pries im Januar 2005 auf dem Weltwirtschaftsforum in Davos seine Politik und insbesondere die Schaffung eines großen Niedriglohnbereichs als großen Erfolg! ${ }^{4}$ Um die Arbeitslosen dazu zu bewegen, verfügbare Arbeitsplätze auch zu geringeren Löhnen anzunehmen, wurden sie von den Agendamaßnahmen unter Druck gesetzt. Diese Politik zielte darauf ab, Arbeitslose zu bewegen, Arbeitsplätze zu jeder Bedingung anzunehmen. Dies wirkte sich auch auf das allgemeine Lohnniveau aus. Schröder pries damit eine Politik, die negierte, dass Beschäftigte von ihrem Einkommen leben können müssen. Dabei hatte schon Smith (1978, 59), der die Bedeutung des Marktes als Funktionsprinzip betonte, auf diese Notwendigkeit hingewiesen. Und Rüstow (2001, 142 f.) äußerte sich 1945 in gleicher Weise.

Die Diagnose, dass Arbeitslosigkeit die Folge von Arbeitsunwilligkeit sei, weist auf ein merkwürdiges Menschenbild hin. Allerdings gab es schon lange Ökonomen, die das als realistisch ansahen und von „freiwilliger" Arbeitslosigkeit spra-

4 „Wir haben einen funktionierenden Niedriglohnsektor aufgebaut, und wir haben bei der Unterstützungszahlung Anreize dafür, Arbeit aufzunehmen, sehr stark in den Vordergrund gestellt" (Schröder, 2005). 
chen. Der österreichische Ökonom Rothschild (1990, 223 ff.) setzte sich 1990 kritisch mit dieser vermeintlichen Ursache der Arbeitslosigkeit auseinander. Dass diese Diagnose nicht zutreffen konnte, ergibt sich schon daraus, dass die Zahl der Arbeitslosen zur Zeit der Agenda ca. zwanzigfach so hoch war wie die Zahl offener Stellen! Selbst wenn alle offenen Stellen hätten besetzt werden können, wäre die Massenarbeitslosigkeit nur graduell vermindert worden (Niemeier, 2010, $320 \mathrm{ff}$.). Es fehlte nicht am Arbeitswillen, sondern an Arbeitsplätzen. Diese fehlten, weil die gesamtwirtschaftliche Nachfrage nicht groß genug war, eine Produktion zu ermöglichen, die zusätzliche Arbeitsplätze hätte entstehen lassen können. Die scheinbaren Erfolge der Agenda sind in Wirklichkeit durch die sich erholende Weltkonjunktur erzielt worden und damit der weltwirtschaftlichen Nachfragedynamik geschuldet. Solow hatte Recht, dass eine steigende gesamtwirtschaftliche Nachfrage die wirtschaftliche Entwicklung fördern und Arbeitslosigkeit vermindern werde. Bofinger (SVR, 2007, 341 ff.; Niemeier, 2010, 320 ff.) wies bereits im Gutachten 2007/2008 nach, dass nicht die Agenda, sondern die Weltkonjunktur für den Abbau der Arbeitslosigkeit verantwortlich war.

\section{Grundrente wird falsch begründet}

Die Grundrente zielt darauf ab, eine „Lebensleistung“ zu belohnen. Wenn entsprechende Leistungen vorliegen, soll die Rente auf ein bestimmtes Niveau angehoben werden. Dafür, dass viele Beschäftigte keine 35 Jahre Beiträge zahlen konnten oder ein zu niedriges Einkommen hatten, war jedoch die Politik verantwortlich. Deshalb ist das Kriterium einer 35-jährigen Beitragszahlung unzulässig. Die Rentner würden damit für die Fehler der Politik bestraft werden. Die Renten müssen aus Steuermitteln auf ein existenzsicherndes Niveau angehoben werden. Die sogenannte Respektrente löst wegen der Vernachlässigung der Ursachen zu geringer Renten weder das Problem des notwendigen Lohnersatzes im Alter noch das der Altersarmut. Sie beabsichtigt auch nicht, die falsch begründete Absenkung des Rentenniveaus rückgängig zu machen. Die politisch bewirkte Rentenminderung, die die Anwendung des Äquivalenzprinzips ausschließt, erfordert ebenso wie die Rentenniveauabsenkung, eine grundlegende Reform des Alterssicherungssystems.

Die notwendige Lösung des Rentenproblems erfordert eine Korrektur der wirtschaftspolitischen Niedriglohnförde- rung und die erneute Gestaltung der Renten als existenzsichernden Lohnersatz. Die vermeintliche Notwendigkeit, eine Lebensleistung in einer Respektrente anzuerkennen, übersieht, dass die angesprochenen Leistungen der Versicherten durch politische Entscheidungen vermindert wurden und deshalb nicht die Ursache der Misere sind. Die Grundrente schließt ferner Gruppen aus, die wegen der Erfüllung gesellschaftlich wichtiger Aufgaben ein niedriges Einkommen erzielten. Diese Probleme müssen dringend gelöst werden, denn „der in der deutschen Alterssicherungspolitik eingeschlagene Weg hat das Potential für eine gesellschaftspolitische Zeitbombe" (Schmähl, 2012, 313).

\section{Literatur}

Althammer, J. W., und H. Lampert (2014), Lehrbuch der Sozialpolitik, 9. Aufl., Springer.

Bofinger, P. (2005), Wir sind besser als wir glauben, $184 \mathrm{f}$.

Dörre, K. (2010), Hartz-Kapitalismus - Vom erfolgreichen Scheitern der jüngsten Arbeitsmarktreformen, in W. Heitmeyer (Hrsg.): Deutsche Zu stände, Folge 9.

Flassbeck, H. und F. Spieker (2007), Das Ende der Massenarbeitslosigkeit, $77 \mathrm{ff}$.

Geyer, J., H. Buslei, P. Gallego-Granados und P. Haan (2019), Anstieg der Altersarmut in Deutschland: Wie wirken verschiedene Rentenreformen?

Hickel, R. (2006), Kassensturz, 172 ff.

Horn, G. A. (2005), Sparwut und Sozialabbau - Die deutsche Krankheit.

Niemeier, E. (2009), Ursachen der deutschen Wachstumsschwäche und Arbeitslosigkeit, Wirtschaftsdienst, 89(9), $592 \mathrm{ff}$.

Niemeier, E. (2010), Hat der Arbeitsmarkt wirklich von Hartz IV profitiert? WSI-Mitteilungen, (6).

Niemeier, E. (2018), Lebensstandardsicherndes Niveau der gesetzlichen Rentenversicherung erforderlich, Wirtschaftsdienst, 98(8), $600 \mathrm{ff}$.

Rothschild, K. W. (1990), Arbeitslose: Gibt's die?, in R. Buchegger, M. Hutter, B. Löderer (Hrsg.), Arbeitslose: Gibt's die? Ausgewählte Beiträge zu ökonomischen und gesellschaftspolitischen Aspekten der Arbeitslosigkeit.

Rüstow, A. (2001), Das Versagen des Wirtschaftsliberalismus, 3. überarbeitete Aufl., Metropolis.

Scherf, W. (2011), Öffentliche Finanzen, 2. Aufl.

Schmähl, W. (2012), Von der Rente als Zuschuss zum Lebensunterhalt zur ,Zuschuss-Rente', Wirtschaftsdienst, 92(5).

Schmähl, W. (2016), Höchste Zeit für einen Ausstieg aus dem Ausstieg“, Wirtschaftsdienst, 96(10), 717.

Schröder, G. (2005), Rede vor dem World Economic Forum am 28. Januar in Davos, https://www.bundesregierung.de/breg-de/service/ bulletin/bulletin-2000-bis-2009/rede-von-bundeskanzler-gerhardschroeder-792094 (29. Juli 2020).

Smith, A. (1978), Der Wohlstand der Nationen, Deutscher Taschenbuch Verlag.

Solow, R. (2007), Die Beschränktheit der makroökonomischen Diskussion überwinden, in R. Schettkat, J. Langkau (Hrsg.), Aufschwung für Deutschland.

SVR (Sachverständigenrat zur Begutachtung der gesamtwirtschaftlichen Entwicklung) (2007), Das Erreichte nicht verspielen, Jahresgutachten 2007/08.

Wyplosz, C. (2007), Deutschland in der Währungsunion, in R. Schettkat, J. Langkau (Hrsg.), Aufschwung für Deutschland.

Title: Pensioners' Poverty Requires Fundamental Reform

Abstract: The German government seeks to improve the situation of longterm pension contributors with the basic pension ("Grundrente"). However, the basic pension only combats the symptoms rather than addressing the causes of low pensions and old age poverty: the policy of low wages and wrong macroeconomic policy. The political responsibility of these pension problems require taxfinanced compensations to guarantee a subsistence level.

JEL Classification: E60, H53, I38, J18 